\title{
latrogenic Pseudoaneurysm of Femoral Artery: Case Report and Literature Review
}

\author{
Martina Lenartova, MD, Department of Cardiology, Marshfield Clinic, Marshfield, Wisconsin \\ Tahir Tak, MD, PhD, Department of Cardiology, Marshfield Clinic, Marshfield, Wisconsin
}

\begin{abstract}
The case of a patient who developed a femoral artery pseudoaneurysm (FAP) following cardiac catheterization is described. It is one of the most troublesome complications after various invasive cardiovascular procedures related to the femoral arterial access site. latrogenic pseudoaneurysms (IPA) form when an arterial puncture site fails to seal, allowing arterial blood to ooze into the surrounding tissues and form a pulsatile hematoma. The FAP occurs in $0.8 \%$ to $2.2 \%$ after interventional procedures. This problem has become more significant due to the exponential growth of interventional cardiology.

Doppler flow mapping has been the mainstay of diagnosis. Diagnostic criteria include: swirling color flow in a mass separate from the affected artery, and a typical "to-and-fro" Doppler waveform in the pseudoaneurysm neck. Ultrasound-guided compression repair has replaced the need for surgical repair of FAP. It has been shown to be a safe and cost-effective method for achieving pseudoaneurysm thrombosis. However, it carries considerable drawbacks including long procedure times, discomfort to patients, high recurrence rate in patients receiving anticoagulant therapy and an overall $3.6 \%$ complication rate.
\end{abstract}

Recently, percutaneous thrombin injection in the FAP has gained popularity despite complications associated with the initial use of high dose thrombin (average dose of 1,100 IU). The technique was refined when low-dose thrombin injections were studied and proved to have the same efficacy and consistently high success rates (average dose used $192 \mathrm{IU}$ ). However, there is a theoretical risk of developing type I IgE-mediated allergic reaction to bovine thrombin.

The indications, advantages, disadvantages, and complications of the various treatment modalities are discussed in this report and review of the literature. Other treatments with collagen injection are also discussed in detail.

REPRINT REQUESTS:

Tahir Tak, MD, PhD

Department of Cardiology

Marshfield Clinic

1000 North Oak Avenue

Marshfield, WI 54449

Telephone: 715-387-5301

Fax: 715-389-4555

Email: tak.tahir@marshfieldclinic.org
KEYWORDS:

Femoral artery; Pseudoaneurysm; Cardiac catheterization; latrogenic complications 


\section{INTRODUCTION}

The femoral artery pseudoaneurysm (FAP) is a troublesome groin complication related to the femoral arterial access site used for invasive cardiovascular procedures. ${ }^{1}$ FAP occur in $0.1 \%$ to $0.2 \%$ of diagnostic angiograms and $0.8 \%$ to $2.2 \%$ following interventional procedures. ${ }^{2}$ With more than one million cardiac catheterizations being performed in the United States each year, post procedure pseudoaneurysm is a relatively rare occurrence. The incidence of FAP has recently increased with the more frequent use of thrombolytics, antiplatelet agents and anticoagulants, and larger-sized cannulas for interventional procedures. ${ }^{1}$

FAPs are usually caused by punctures of the femoral artery which are too distal, that is, at the level of bifurcation of the femoral artery or below. ${ }^{1}$ Iatrogenic pseudoaneurysms (IPA) form when an arterial puncture site fails to seal, allowing arterial blood to jet into the surrounding tissues and form a pulsatile hematoma. ${ }^{3}$ These lesions lack a fibrous wall and are contained by a surrounding shell of hematoma and the overlying soft tissues. It can present as a new thrill or bruit, pulsatile hematoma, or marked pain or tenderness.

Complications of pseudoaneurysms include rupture, distal embolization, local pain, neuropathy and local skin ischemia. ${ }^{3}$

\section{CASE REPORT}

A patient, 82 years of age, was admitted with exertional chest pain associated with shortness of breath. A sestamibi nuclear scan showed apical ischemia and left ventricular dysfunction on the left ventricular angiogram. Pertinent medical history included end-stage renal disease needing hemodialysis, non-insulin dependent diabetes mellitus, poorly controlled hypertension and chronic obstructive pulmonary disease. The patient underwent cardiac catheterization. This revealed $80 \%$ stenosis of the mid-left anterior descending, $40 \%$ stenosis of the intermediate ramus and distal circumflex, and a dominant right coronary artery without significant stenosis. Percutaneous transluminal coronary angioplasty (PTCA) with stent placement to the mid-left anterior descending was performed with no residual stenosis with Thrombolysis In Myocardial Infarction (TIMI)-3 flow seen in the left anterior descending after the procedure. Dialysis was performed on the day of cardiac intervention and the patient was discharged in stable condition.

Six days following discharge from the hospital, the patient experienced severe sharp pain in the right groin without any skin discoloration of the right lower extremity. The patient denied numbness, tingling, or loss of voluntary movement.
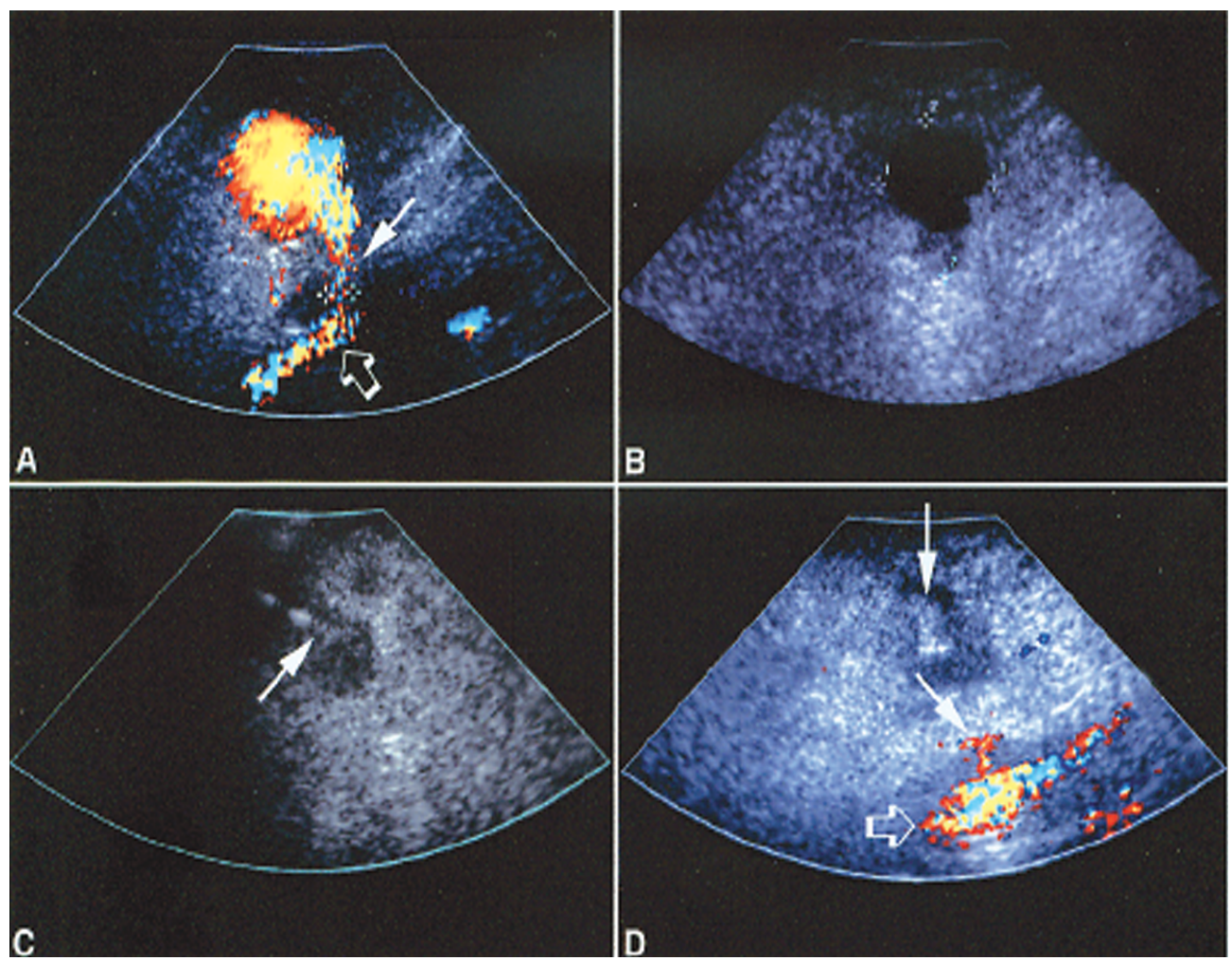

\section{Figure 1.}

A. Color flow demonstrating blood flow in right femoral artery (open arrow) through the neck into the pseudoaneurysm.

B. Pseudoaneurysm without superimposition of color flow.

C. Needle entering pseudoaneurysm.

D. Top arrow pointing to the coagulation of blood after thrombin injection. Mid-arrow points to the neck of the pseudoaneurysm. The open arrow points to antegrade blood flow in the femoral artery. 
On physical examination, a pulsatile mass was noted in the right groin. Ultrasound confirmed a right FAP (figure 1) which was treated with thrombin injection into the cavity of the pseudoaneurysm. With the guidance of ultrasound, 200 IU of thrombin $(0.2 \mathrm{~mL}$ of $1,000 \mathrm{IU}$ in $1 \mathrm{~mL}$ diluting solution) was injected. The pseudoaneurysm was noted to clot after the procedure (figure 1D). An ultrasound repeated the following day showed a thrombosed cavity of the pseudoaneurysm with just a few scattered subcutaneous hematomas identified in the right groin region. No new pseudoaneurysmal formation was identified.

\section{DISCUSSION}

Duplex scanning, along with pulsed and color Doppler flow mapping has been the mainstay in diagnosing FAP. Criteria used to diagnose a pseudoaneurysm include: swirling color flow seen in a mass separate from the affected artery, color flow within a tract leading from the artery to the mass consistent with pseudoaneurysm neck, and a typical "to and fro" Doppler waveform in the pseudoaneurysm neck. ${ }^{3}$

Several therapeutic strategies have been developed to treat this complication. They include ultrasound-guided compression repair (UGCR), surgical repair, and minimally invasive percutaneous treatments (thrombin injection, coil embolization and insertion of covered stents). ${ }^{1}$

UGCR has become the first-line treatment of pseudoaneurysms at many institutions. The introduction of this method in 1991 by Fellmeth et al. ${ }^{4}$ has significantly reduced the need for surgical repair of FAP. It has been shown to be a safe and cost-effective method for achieving pseudoaneurysm thrombosis. ${ }^{3}$ The relative cost of percutaneous thrombin injection (\$695) is much lower than open vascularization $(\$ 3,772)$, endovascular repair with stent placement $(\$ 2,542)$, or UGCR $(\$ 1,223)$. However, UGCR has considerable drawbacks including long procedure time, discomfort to patients and a relatively high recurrence rate in patients receiving anticoagulant therapy (as high as $25 \%$ to $35 \%$ ). ${ }^{3}$ UGCR has been shown to be less successful in patients with large FAP (i.e., larger than $3 \mathrm{~cm}$ to $4 \mathrm{~cm}$ in diameter) and those who cannot tolerate the associated discomfort. 5 The procedure carries an overall complication rate of $3.6 \%$ and risk of rupture of $1 \%{ }^{3}$ Complications include acute pseudoaneurysmal enlargement, frank rupture, vasovagal reactions, deep vein thrombosis, atrial fibrillation and angina. ${ }^{3}$ Moreover, UGCR requires the availability of an ultrasound device and the presence of skilled personnel during the procedure. The technique involves applying compression on the pseudoaneurysm neck with the ultrasound transducer until the flow within the neck is obliterated. Pressure is applied for a period of 1 minute, with the precedure repeated 10 times. At the end of each period compression is released briefly to assess pseudoaneurysm patency and to reposition the transducer. Care must be taken to avoid compromising flow within the underlying femoral artery. After successful thrombosis patients should be kept supine for a few hours, with the affected leg in the stretched position.
Contraindications to this technique include inaccessible site, limb ischaemia, infection, large hematomas with overlying skin ischaemia, compartment syndrome and prosthetic grafts. ${ }^{5}$

In 1986 Cope and Zeit ${ }^{6}$ and subsequently in 1987 Walker et al. ${ }^{7}$ first introduced the technique of percutaneous thrombin injection in the FAP. The technique would not gain popularity for another ten years. The first attempts to close a FAP with ultrasound-guided thrombin injections were prone to several complications. Cases of distal migration of the thrombin were reported. ${ }^{8}$ Thrombin is used in liquid form which is why it can diffuse from the pseudoaneurysm through the neck toward the lumen of the femoral artery. ${ }^{1}$ It is also possible that if the thrombin is injected in a very diluted concentration, it may not remain in the cavity of the FAP long enough to form a clot. ${ }^{1}$ The average dose of thrombin utilized in the literature is approximately $1,100 \mathrm{IU}$, with a range ${ }^{9}$ of 100 to $5,000 \mathrm{IU}$. In addition, it has been described that patients receiving thrombin are at risk for developing anti-factor $\mathrm{V}$ antibodies, ${ }^{1}$ which may expose them to an immunologic cross-reaction to human factor $\mathrm{V}$.

Reeder et al. introduced a new method of low-dose thrombin injection for the treatment of pseudoaneurysms. ${ }^{9}$ An average dose of $192 \mathrm{U}$ of thrombin was used (5-fold lower than previously reported) in 23 patients with 26 postcatheterization FAPs. Time to coagulation ranged from 10 to 60 seconds. There were no complications. The investigators were able to successfully thrombose all pseudoaneurysms, even in the presence of anticoagulation. Their study demonstrated that a much smaller dose of a potentially dangerous medication can achieve the same efficacy as previously used higher doses.

Compared with surgical repair, treatment of pseudoaneurysms with thrombin injection offers many advantages. The success rate of thrombin injection reported in the literature has been consistently high, at an average of $97 \%$, even with patients treated with therapeutic levels of anticoagulants. Treatment can usually be completed within several minutes.

Theoretically, a lower dose of a potentially dangerous medication should decrease the risks associated with its use. As more reports are published, it is possible more complications will come to light. In addition to distal limb ischemia caused by in situ thrombosis from thrombin leakage, there is a hypothetical risk of type I IgE-mediated allergic reaction to bovine thrombin. Topical bovine thrombin has been used extensively for hemostasis for more than 20 years. Recent descriptions of antibody responses ${ }^{2}$ show high titers against endogenous coagulation factors, with resulting bleeding complications. Prior exposure to bovine thrombin is considered a contraindication to treatment of pseudoaneurysms with thrombin by some investigators. 
At the health center in Utrecht and Nieuwegein in the Netherlands, collagen injection as a method for FAP closure was developed as an alternative choice for either primary treatment of the iatrogenic pathology or treatment in the majority of cases where UGCR was unsuccessful. ${ }^{1}$ The investigators studied the method in 110 patients with FAP following catheterization procedures $(51 \%$ diagnostic catheterization, $42.8 \%$ interventional procedures, $6 \%$ other cardiac procedures). Purified bovine collagen in the form of a biodegradable adhesive paste was injected to promote hemostasis and accelerate the clotting process within the pseudoaneurysm. The success rate in this study was $98.2 \%$ (109 of 110 patients). The results of this study rank collagen injection among the most successful minimally invasive percutaneous FAP closure techniques.

Purified bovine collagen has been used in surgical procedures as an adjunct to hemostasis when control of bleeding by ligature or other conventional methods remains ineffective. When collagen comes in contact with blood, platelets aggregate on the collagen and release coagulation factors that, together with plasma factors, result in the formation of a fibrin matrix. Once implanted into tissues of an organism, collagen is ultimately degraded and progressively resorbed by granulocytes and macrophages. Contraindication to this procedure includes suspected underlying infection and known allergy to collagen or beef products.

Injection of collagen is easily accepted by the patients as there is no need for systemic analgesic medication during the procedure. Complete obliteration of the pseudoaneurysm is usually achieved within 10 seconds, which is comparable to that with thrombin. The coagulation status of the patient does not preclude its use.

The advantages of the collagen lie in its physicochemical properties. The fact that it consists of long paste fibers allows the collagen to remain within the FAP cavity, which putatively reduces the risk of migration through the neck of the FAP, or through a fistula. In conclusion, the technique of collagen injection is a fast, expeditious method, permitting early mobilization and discharge.
Other non-operative methods of treating pseudoaneurysms include placement of covered stents/endoluminal prostheses. Majority of the prostheses reported in the literature have been used for the exclusion of atherosclerotic aneurysms. A few reports have focused on the use of percutaneous coil placement (stents) to occlude the FAP. In some cases the coil was placed in the neck, while in other patients the coil was placed inside the pseudoaneurysm in order to achieve closure and local thrombosis. ${ }^{10,11}$ Contraindications to the use of endovascular prosthesis in the management of IPA are injection, smaller arteries $(<7 \mathrm{~mm})$ and the essential parent artery, which needs to be saved.

Loose et al. ${ }^{12}$ describe a method of percutaneous injection of fibrin adhesive. This technique involves percutaneous injection of the adhesive components using ultrasound and screening control following successful occlusion of the aneurysm neck by angioplasty balloon. ${ }^{12}$ The fibrin adhesive mimics the final stage of coagulation cascade.

Thrombin is used to convert fibrinogen to fibrin, and the fibrin is then crosslinked by factor XIIIa in the presence of calcium to create a mechanically stable network filling the aneurysm. Tissue adhesives based on fibrinogen, thrombin, and factor XIII have been commercially available for many years. Fibrin has been extensively used in neurosurgery for repairing cerebrospinal leaks, sealing vascular anastomotic sites, reinforcing aneurysm clips and for hemostasis after tumor resection. The technique has several advantages-the balloon virtually eliminates the chance of distal embolization and decreases the likelihood of the tissue adhesive having any effects on the patient's coagulation status. The tissue adhesive does not rely on the patient's own clotting factors. Therefore it is more likely to work in anticoagulated patients.

Due to the procedural simplicity, ultrasound-guided injection of thrombin remains a very appealing treatment to most physicians. Currently, research efforts are directed at developing a sponge-like application form of collagen. Then small quantities of sponge-like collagen would suffice to obliterate the FAP cavity, which could be monitored by ultrasound.

Table 1. Indications for surgical treatment of femoral artery pseudoaneurysm.

Rapid expansion of the IPA. There may not be time to wait for noninvasive treatments.

Concomitant distal ischemia or neurological deficit due to local pressure from the IPA, or distal embolization from within it.

"Mycotic" infection of IPA. (The natural history is then one of rapid enlargement with subsequent rupture or septic emboli. Embolization with foreign bodies will only increase infection and is contraindicated.)

Failure of percutaneous intervention.

Compromised soft tissue viability. 
It is unfortunate that most pseudoaneurysms occur in patients least tolerant to general anesthesia, vascular reconstruction and associated blood loss. Treatment by UGCR or percutaneous embolization is an attractive option and probably the first choice at many institutions for these reasons. However, there are situations when surgical treatment may be necessary. These are listed in table 1.

Discussion of surgical approach in a particular situation is beyond the scope of this summary. Surgery is usually effective and definitive. Nevertheless, it is also a relatively expensive means of IPA repair. Although costs of the various procedures utilized for closure of FAP will vary between institutions, an approximate figure after each intervention in the midwestern United States has been provided. Please be aware that these costs may change rapidly with time.

\section{ACKNOWLEDGMENTS}

The authors wish to thank Marshfield Clinic Research Foundation for its support though the assistance of Alice Stargardt in the preparation of this manuscript.

\section{REFERENCES}

1. Hamraoui K, Ernst SM, van Dessel PF, Kelder JC, ten Berg JM, Suttorp MJ, Jaarsma W, Plokker TH. Efficacy and safety of percutaneous treatment of iatrogenic femoral artery pseudoaneurysm by biodegradable collagen injection. J Am Coll Cardiol 2002;39:1297-1304.

2. Messina LM, Brothers TE, Wakefield TW, Zelenock GB, Lindenauer SM, Greenfield LJ, Jacobs LA, Fellows EP, Grube SV, Stanley JC. Clinical characteristics and surgical management of vascular complications in patients undergoing cardiac catheterization: interventional versus diagnostic procedures. J Vasc Surg 1991;13:593-600.

3. Eisenberg L, Paulson EK, Kliewer MA, Hudson MP, DeLong DM, Carroll BA. Sonographically guided compression repair of pseudoaneurysms: further experience from a single institution. AJR Am J Roentgenol 1999;173:1567-1573.

4. Fellmeth BD, Roberts AC, Bookstein JJ, Freischlag JA, Forsythe JR, Buckner NK, Hye RJ. Postangiographic femoral artery injuries: nonsurgical repair with US-guided compression. Radiology 1991;178:671-675.

5. O’Sullivan GJ, Ray SA, Lewis JS, Lopez AJ, Powell BW, Moss AH, Dormandy JA, Belli AM, Buckenham TM. A review of alternative approaches in the management of iatrogenic femoral pseudoaneurysms. Ann R Coll Surg Engl 1999;81:226-234.

6. Cope C, Zeit R. Coagulation of aneurysms by direct percutaneous thrombin injection. AJR Am J Roentgenol 1986;147:383-387.

7. Walker TG, Geller SC, Brewster DC. Transcatheter occlusion of a profunda femoral artery pseudoaneurysm using thrombin. AJR Am J Roentgenol 1987;149:185-186.

8. Lennox A, Griffin M, Nicolaides A, Mansfield A. Regarding "Percutaneous ultrasound guided thrombin injection: a new method for treating postcatheterization femoral pseudoaneurysms." J Vasc Surg 1998;28:1120-1121.

9. Reeder SB, Widlus DM, Lazinger M. Low-dose thrombin injection to treat iatrogenic femoral artery pseudoaneurysms. AJR Am J Roentgenol 2001;177:595-598.

10. Pan M, Medina A, Suarez de Lezo J, Romero M, Hernandez E, Segura J, Melian F, Wanguemert F, Landin M, Benitez F, Amat M. Obliteration of femoral pseudoaneurysm complicating coronary intervention by direct puncture and permanent or removable coil insertion. Am J Cardiol 1997;80:786-788.
11. Saito S, Arai H, Kim K, Aoki N, Tsurugida M. Percutaneous transfemoral spring coil embolization of a pseudoaneurysm of the femoral artery. Cathet Cardiovasc Diagn 1992;26:229231.

12. Loose HW, Haslam PJ. The management of peripheral arterial aneurysms using percutaneous injection of fibrin adhesive. Br J Radiol 1998;71:1255-1259. 